
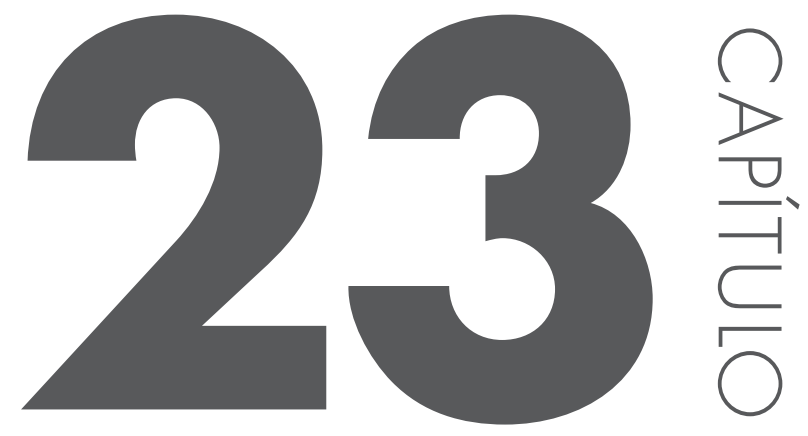

\title{
ENSINANDO GEOMETRIA ANALÍTICA SUSTENTADO POR PROGRAMAC̣ÃO LINEAR
}

Souza Neto, André Luis de ';

Queiroz, Thiago Alves de ${ }^{2}$

1 Unidade de Matemática e Tecnologia, Universidade Federal de Goiás - Regional Catalão

2 Unidade de Matemática e Tecnologia, Universidade Federal de Goiás - Regional Catalão

* email: andrelvisneto@gmail.com 
Resumo: A Geometria Analítica tem sido motivo de muita angústia entre os alunos do ensino médio. Dessa angústia nasce a vontade de tornar o assunto mais acessível aos alunos e, para isso, a busca de exemplos reais vividos no dia a dia destes se torna relevante. Este trabalho tem como objetivo facilitar a relação dos alunos com alguns dos tópicos da Geometria Analítica. Para tanto, o uso da programação linear e de um software matemático se tornam uma metodologia importante, pois, apoiando-se neles surgem possibilidades que contribuem para o alcance dos seguintes objetivos: lidar com a modelagem, resolver problemas do cotidiano dos alunos e interpretar resultados obtidos em cenários práticos na geometria analítica.

\section{Palavras-chave: Programação linear; Ensino médio; Geometria Analítica}




\section{Physics in the Elementary School: the water-drop microscope}

Abstract: This paper presents an application in classroom of physics teaching at elementary school through an interdisciplinary and experimental approach. Classes were taught to students in the program Mais Educação and Education for Youth and Adults (EJA - Educação de Jovens e Adultos), the city of Goiânia, state of Goiás. Interdisciplinary practice, investigative and experimental lessons. Considering the importance of interdisciplinary practice and investigative and experimentation lessons, we taught four classes, where concepts of Physics, Geography and Biology were approached. In the first lesson, concepts of lenses and refraction were addressed; in the second lesson water in streams in the region were collected in an field work activity; in the third the subjects were siltation, riparian forest, permanent preservation area and respect to the environment and in the last lesson the water-drop microscope was built, when it was possible to view the expanded shadow of the microorganisms present in the water droplet. Was remarkable the interest and participation of students in class, making them active elements in the teaching-learning process.

\section{Keywords: Physics Teaching; Mindstorms NXT; Robotics .}

\section{Referências bibliográficas}

AZEVEDO, M.A.R.; ANDRADE, M.F.R.. O conhecimento em sala de aula: a organização do ensino numa perspectiva interdisciplinar, Educar em Revista, Curitiba, n.30, 2007.

BRASIL. Lei No 9.394, de 20 de dezembro de 1996. Lei de Diretrizes e Bases da Educação Nacional. Diário Oficial da União, Brasilia, 23 de dez 1996. p. 27833.

BRASIL. Parâmetros Curriculares Nacionais para o Ensino Fundamental: Matemática, Secretaria de Educação Fundamental. Brasília: MEC/SEF, 1998.

CARVALHO, A.M.P. Ciências no Ensino Fundamental. Cad Pesq, n. 101, 1997

FAZENDA, I.C.A.. Interdisciplinaridade: um Projeto em Parceria. São Paulo: Loyola, 2002.

HEWITT, P.G. Física Conceitual. Porto Alegre: Bookman, 2011 
PLANINSIC, G. Water-Drop Projector. Phys Teach, v.39, n.2, 2001.

ROSA, C.T.W.; ROSA, Á.B. Ensino experimental de física na universidade de passo fundo. In: Encontro nacional de pesquisa em ensino de física, 9, 2004, Jaboticatubas, MG

ROZA, J.P. A pesquisa no processo de formação de professores: intenções e experiências docentes e discentes e as limitações deste exercício - um olhar sob duas realidades educacionais. Porto Alegre: 2005. Disponível em: <https://www.lume.ufrgs.br> Acesso em: 09 de novembro de 2014.

THIESEN, J.S. A interdisciplinaridade como um movimento articulador no processo ensinoaprendizagem. Revista Brasileira de Educação, v. 13, n.39, 2008

VILLANI, A. Ideias espontâneas e ensino de Física. Revista Brasileira de Ensino de Física, vol. 11 , 1989. 


\section{Introduc̣ão}

O trabalho ora proposto surge da necessidade de buscar outras possibilidades que possam contribuir para melhorar o ensino-aprendizado dos alunos do ensino médio em geometria analítica. $O$ trabalho tem como eixo o seguinte questionamento: Como o uso da programação linear poderá auxiliar o ensino da geometria analítica no ensino médio?

O objetivo geral é compreender o motivo da matemática, em particular, da geometria analítica ser considerada, entre os discentes, como uma disciplina difícil. Diante desse objetivo geral, o objetivo específico é propor, por meio da programação linear combinada com o uso 
de um software matemático, uma nova maneira de ensinar geometria analítica para os alunos do ensino médio.

A hipótese que levantamos para a pergunta proposta é que, relacionando o cotidiano dos alunos aos conteúdos de sala de aula, seria possível melhorar o ensino-aprendizagem desses alunos tornando o ensino da geometria analítica mais eficaz por meio de exemplos do dia a dia, e envolvendo a programação linear como suporte.

De acordo com Dewey (1967, p. 22): "Com efeito, sendo a educação o resultado de uma interação, através da experiência, do organismo com o meio ambiente, a direção da atividade educativa é intrínseca ao próprio processo da atividade". Segundo os Parâmetros Curriculares Nacionais no Ensino Médio (2006), ao aluno do ensino médio deve ser possibilitado, de forma combinada, o desenvolvimento de práticas em que o conhecimento venha contextualizado, e de conhecimentos mais amplos e abstratos.

A pesquisa operacional teve início no Brasil na década de 1960, sendo criada, posteriormente, a SOBRAPO (Sociedade Brasileira de Pesquisa Operacional), que publica periódicos científicos há mais de 25 anos no assunto. Para Hillier e Lieberman (2013), a pesquisa operacional visa resolver problemas que estão relacionados à condução e coordenação das operações dando à natureza das organizações uma característica secundária, por isso pode ser usada em diversas áreas.

A pesquisa operacional apresenta subdivisões incluindo a programação linear (PL), a programação inteira (PI) e a programação dinâmica. $\mathrm{O}$ interesse diz respeito à $\mathrm{PL}$, uma vez que esta é uma técnica que se preocupa em minimizar ou maximizar um certa função, chamada objetivo, considerando restrições a essa função. Além disso, as equações e inequações utilizadas nesse processo são de primeiro grau. Para tornar o assunto mais significativo para os alunos do ensino médio, aborda-se apenas equações e inequações com duas ou três variáveis.

De acordo com os Parâmetros Curriculares Nacionais do Ensino Médio (2006), que orienta o ensino médio no Brasil, um dos objetivos é combinar o conhecimento para obter um desenvolvimento mais amplo do mundo. Dessa forma, é missão do professor trazer o cotidiano do aluno para a sala de aula e dar aos estudantes a oportunidade de aprender novos conteúdos e aprofundar os já existentes em seu leque de conhecimentos.

O trabalho está organizado da seguinte maneira: a Seção 2 apresenta a programação linear, incluindo as questões para a construção de modelos na forma padrão e a obtenção da solução pelo método gráfico; o estudo envolvendo o uso de um software matemático é apresentado na Seção 3; e, os comentários finais e algumas direções para outros trabalhos são dados na Seção 4.

\section{Um Pouco de Programação Linear}

A seguir, faz-se a enunciação de um problema típico que, segundo Yoshida (1987), aparece em indústrias de produção de ração animal. A ideia é mostrar quais etapas o aluno deve seguir para construir o modelo de programação linear. $O$ professor deve trabalhar com os alunos buscando motivação por meio de exemplos que aconteçam no dia a dia dos mesmos. 
Situação-problema: Uma jovem necessita ingerir todos os dias pelo menos $20 \mathrm{~g}$ de uvas passas, pelo menos $10 \mathrm{~g}$ de castanha do Pará e, pelo menos, $2 \mathrm{~g}$ de óleo de cártamo. Esses ingredientes são encontrados em dois tipos de alimentos, o biscoito A e o biscoito $B$. As quantidades desses ingredientes em cada biscoito são apresentadas na Tabela 1, assim como o preço unitário de cada biscoito. A jovem deseja comprar esses produtos pelo menor preço possível. Diante disso, faça o modelo para determinar as quantidades de cada biscoito que a jovem deve comprar.

Tabela 1: Relação entre os biscoitos para a situação-problema.

\begin{tabular}{|l|c|c|c|c|}
\cline { 2 - 5 } \multicolumn{1}{c|}{} & $\begin{array}{c}\text { Uva } \\
\text { passa }\end{array}$ & $\begin{array}{c}\text { Castanha do } \\
\text { Pará }\end{array}$ & $\begin{array}{c}\text { Óleo de } \\
\text { cártamo }\end{array}$ & $\begin{array}{c}\text { Preço Unitário } \\
\text { em R } \$\end{array}$ \\
\hline Biscoito A & 4 & 1 & 1 & 30 \\
\hline Biscoito B & 1 & 2 & - & 20 \\
\hline
\end{tabular}

Fonte: Elaborado pelo autor

O primeiro passo da modelagem é descobrir as variáveis de decisão, neste caso, são a quantidade de biscoito $A$ (representada pela variável $x$ ) e a quantidade de biscoito $B$ (representada pela variável y). A partir da informação de que o biscoito $A$ custa 30 reais a unidade e o biscoito $B$ custa 20 reais a unidade, escreve-se a função objetivo:

Minimizar $\mathrm{z}=30 \mathrm{x}+20 \mathrm{y}$

Se uma unidade do biscoito A fornece 4 unidades de uvas passas e, da mesma forma, o biscoito $B$ fornece 1 unidade de uvas passas, tem-se a primeira restrição em relação às uvas passas, pois devem ser ingeridas pelo menos 20 unidades, ou seja:

$4 x+y \geq 20$

Da mesma forma, quanto à castanha do Pará, o biscoito A fornece 1 unidade e o biscoito $B$ fornece 2 unidades. Assim, tem-se a segunda restrição em relação à castanha do Pará, devendo ser ingeridas, pelo menos, 10 unidades, ou seja:

$x+2 y \geq 10$

Analogamente, há uma restrição para o óleo de cártamo, mas, observando-se que apenas o biscoito A possui este componente, chegando em:

$x \geq 2$

Assim, o problema fica formulado, isto é, modelado matematicamente por:

Minimizar $\mathrm{z}=30 \mathrm{x}+20 \mathrm{y}$ 
sujeito a: $\left\{\begin{array}{c}4 x+y \geq 20 \\ x+2 y \geq 10 \\ x \geq 2 \\ x \geq 0, y \geq 0\end{array}\right.$

\subsection{A Resolução pelo Método Gráfico}

A resolução gráfica de um problema de programação linear no ensino médio está ligada ao ensino da geometria analítica no que tange às equações da reta e inequações. Assim, apresenta-se adiante os conteúdos relacionados à resolução de problemas desse tipo, baseando-se em: Reis e Silva (1996), Silva e Barreto Filho (2000) e Dante (2005).

O problema de programação linear tem apenas duas variáveis de decisão, com isso o mesmo pode ser resolvido usando o método gráfico (PASSOS, 2008). O método gráfico possui uma vantagem didática muito grande, que é permitir ao aluno do ensino médio a visualização da lógica que acompanha a solução, para, posteriormente, compreender métodos mais avançados de resolução, como por exemplo, o método simplex.

$\mathrm{Na}$ resolução de um problema de programação linear existe a garantia de que a solução está em um dos vértices quando a região viável é delimitada (ARENALES et al., 2007). Com isso, após desenhar a região, o próximo passo é encontrar as coordenadas dos vértices, que são obtidas transformando as inequações em equações. Em seguida, calcula-se a interseção entre as equações para obter os vértices de interesse.

Segundo Dantzig (1963, p. 60), se a região viável de um problema de programação linear é não vazia, então, a função objetivo atinge o valor máximo ou o valor mínimo nos pontos extremos desta região. Por outro lado, se a região viável é ilimitada, a função objetivo pode ou não assumir valores máximos ou mínimos. Se atingir um valor máximo ou mínimo, este certamente ocorrerá em algum ponto extremo.

A partir disso, uma função objetivo $\mathrm{z}=\mathrm{ax}+$ by deve atingir valores extremos na região $R$ do plano cartesiano, em que $R$ é formada pela intersecção das inequações que são as restrições do problema. Para cada valor que $z$ assumir, tem-se uma reta paralela à anterior, de forma que se pode "fatiar" a região $R$ em diversas retas paralelas, ou seja, desenhar as curvas de nível da função objetivo. Sendo R fechada e limitada, é possível obter uma curva de nível, definida pelas variáveis $x$ e $y$, que atinge um dos vértices extremos da região. A Figura 2 traz a região viável da Figura 1 , que é delimitada pelos pontos $A, B, C, D$ e $E$, sendo que cada reta tracejada representa a função $\mathrm{z}=4 \mathrm{x}+6 \mathrm{y}$ para um valor diferente de $\mathrm{z}$.

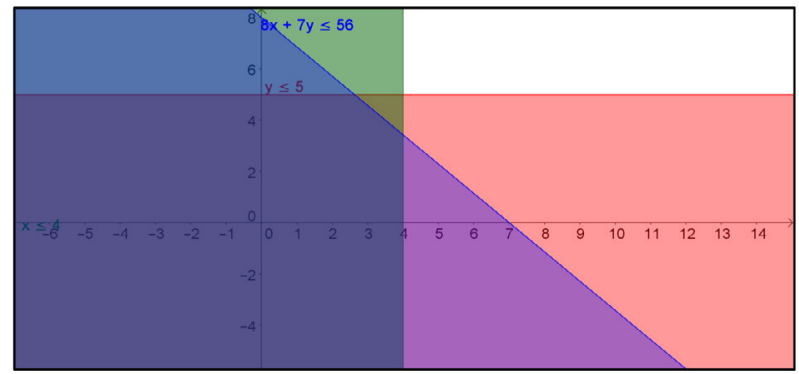

Figura 1: Região viável com seus pontos extremos A, B, C, D e E. Fonte: Elaborada pelo autor 


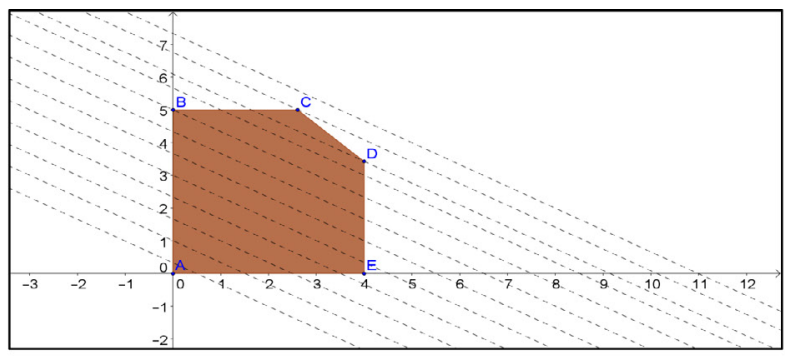

Figura 2: Região viável da Figura 1 sendo interceptada pela função objetivo.

Fonte: Elaborada pelo autor

\subsection{Determinando os Pontos Extremos}

Para trabalhar com a resolução pelo método gráfico, o aluno deve ser capaz, em um primeiro momento, de fazer a modelagem, ou seja, transformar o problema dado em equações e inequações matemática. Após a modelagem do problema, parte-se para o uso dos conceitos vistos em sala de aula ao estudar geometria analítica. Neste ponto, o aluno necessita ilustrar cada uma das restrições para obter a região viável.

Os pontos extremos de uma região viável, que estão relacionados com a solução ótima, caso a mesma exista, são obtidos por meio da interseção entre as equações que, por sua vez, são obtidas das inequações. Para obter as equações, troca-se a relação de desigualdade por uma igualdade em cada restrição do problema de programação linear.

A partir da obtenção dos pontos extremos, o aluno tem duas situações: (i) caso a região viável seja limitada, os pontos extremos são substituídos na função objetivo e aquele que atende ao critério de otimização (maximizar ou minimizar) é, então, considerado como solução ótima do problema; ou, (ii) se a região for ilimitada, o aluno precisa atentar para as curvas de nível para ver se existe solução ótima para o problema, ou se o problema é ilimitado.

Para tanto, considere a situação-problema sobre as quantidade de biscoitos $A$ e $B$ que a jovem deve comprar, com o objetivo de minimizar o gasto na compra dos biscoitos. Após ter modelado o problema, cujo modelo se encontra na eq. (5), transformando-o em equações e inequações matemáticas, o professor deve solicitar aos alunos para colocaram no plano Cartesiano as inequações, conforme ilustra a Figura 3. Então, proceder com a delimitação da região viável do problema e a determinação dos pontos extremos, conforme mostra a Figura 4.

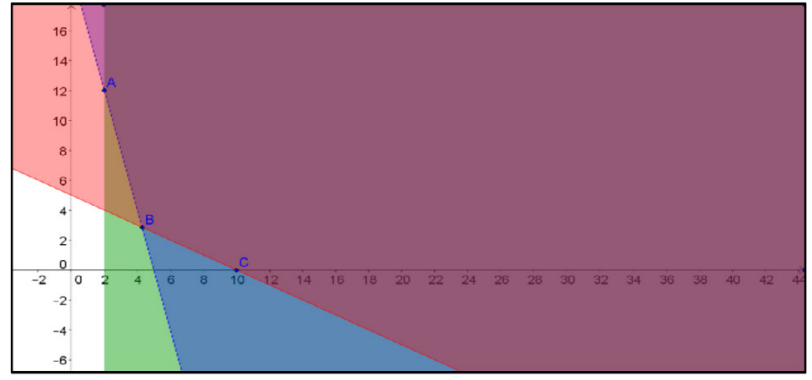

Figura 3: Representação das inequações do modelo (5).

Fonte: Elaborada pelo autor 


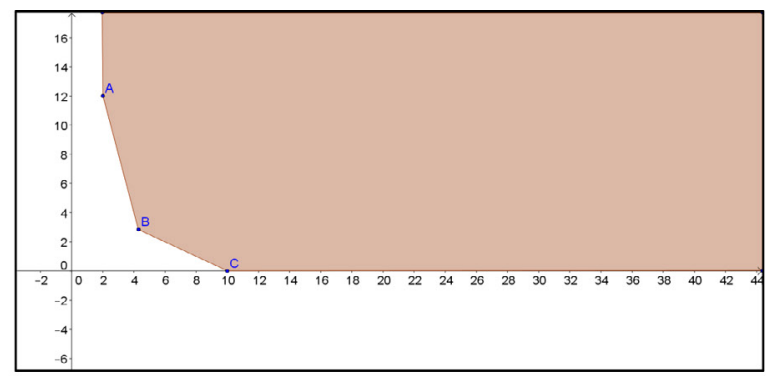

Figura 4: - Representação da região viável do modelo (2.6)

Fonte: Elaborada pelo autor

Para determinar os pontos extremos na Figura 4, toma-se cada uma das restrições, incluindo as de não-negatividade, e faz-se a interseção entre elas. $O$ ponto $A$ é obtido pela interseção da reta $4 \mathrm{x}+\mathrm{y}=20 \mathrm{com} \mathrm{x}=2$. O aluno precisa substituir $\circ$ valor de $\mathrm{x}=2$ na primeira equação e resolver para obter como resultado $y=12$. Com isso, o vértice $A$ possui coordenadas $(2,12)$. Similarmente, obtém-se os vértices $B$ e $C$, de forma que Tabela 2 sumariza as coordenadas e interseções que foram resolvidas.

Tabela 2: Relação de pontos extremos e coordenadas para o modelo (5).

\begin{tabular}{|c|c|c|}
\hline Ponto Extremo & Interseção & Coordenadas $(\mathrm{x} ; \mathrm{y})$ \\
\hline $\mathrm{A}$ & $\left\{\begin{array}{c}4 \mathrm{x}+\mathrm{y}=20 \\
\mathrm{x}=2\end{array}\right.$ & $(2 ; 12)$ \\
\hline $\mathrm{B}$ & $\left\{\begin{array}{c}\mathrm{x}+2 \mathrm{y}=10 \\
4 \mathrm{x}+\mathrm{y}=20\end{array}\right.$ & $(4,29 ; 2,86)$ \\
\hline $\mathrm{C}$ & $\left\{\begin{array}{c}\mathrm{x}+2 \mathrm{y}=10 \\
\mathrm{y}=0\end{array}\right.$ & $(10 ; 0)$ \\
\hline
\end{tabular}

Fonte: Elaborada pelo autor.

Partindo da Tabela 2, o aluno deve observar que se busca a minimização, de forma que um dos vértices extremos pode corresponder a solução ótima. $O$ próximo passo consiste na substituição dos vértices $A, B$ e $C$ na função objetivo. A Tabela 3 traz os resultados obtidos para a função objetivo do modelo (5) após a substituição dos vértices.

Tabela 3: Pontos extremos substituídos na função objetivo do modelo (25)

\begin{tabular}{|c|c|c|}
\hline $\begin{array}{c}\text { Ponto } \\
\text { Extremo }\end{array}$ & Solução $(\mathrm{x} ; \mathrm{y})$ & Função objetivo $\mathrm{z}=30 \mathrm{x}+20 \mathrm{y}$ \\
\hline $\mathrm{A}$ & $(2 ; 12)$ & $\mathrm{z}=30 \times 2+20 \times 12=300$ \\
\hline $\mathrm{B}$ & $(4,29 ; 2,86)$ & $\mathrm{z}=30 \times 4,29+20 \times 2,86=185,9$ \\
\hline C & $(10 ; 0)$ & $\mathrm{z}=30 \times 10+20 \times 0=30$ \\
\hline
\end{tabular}

Fonte: Elaborada pelo autor. 
A partir dos resultados apresentados na Tabela 3, o aluno precisa se atentar para o critério de otimização do problema em estudo. No caso do modelo (5), deseja-se a solução que minimiza o gasto, resultando no vértice $(x, y)=(10,0)$ e valor objetivo igual a 30 .

\section{Usando um Software para o Ensino de Geometria}

O Geogebra é um aplicativo (software) de matemática dinâmica que proporciona uma combinação entre conceitos da geometria analítica e da álgebra. Uma das primeiras aplicações do programa é na construção de pontos, retas, segmentos de retas, polígonos, etc. Ainda permite fazer a plotagem de gráficos de equações em duas dimensões e também lidar com vetores, derivar, integrar funções, encontrar raízes de equações e pontos extremos de uma função (HOHENWARTER; HOHENWARTER, 2009).

Por ser um aplicativo didaticamente simples e um software livre, que pode ser usado nos sistemas operacionais Windows e Linux, tem-se no Geogebra uma ferramenta importante para auxiliar nas aulas do ensino médio. Por esse motivo, fez-se a sua escolha para propiciar o ensino da programação linear, juntamente com a geometria analítica.

Nesse trabalho, como o aluno precisa lidar com a região viável relacionada ao modelo de programação linear, faz-se necessário usar a entrada de comandos. Por meio dela, o aluno escreve cada uma das inequações (das respectivas restrições) e o Geogebra cria a ilustração na zona gráfica.

Visando auxiliar o aluno no trabalho com o Geogebra, o professor pode tomar qualquer modelo com duas variáveis de decisão, uma vez que a representação ocorrerá no plano cartesiano. A primeira observação é a troca das variáveis $\mathrm{x}_{1}$ por $\mathrm{x}$ e $\mathrm{x}_{2}$ por y caso o modelo assim esteja representado, uma vez que essa troca facilitará o entendimento do aluno. Vale destacar que no decorrer da educação básica os professores geralmente usam as variáveis $\mathrm{x}$ e y. Diante disso, considere o seguinte modelo de programação linear:

Maximizar $z=20 x+24 y$
sujeito a: $\left\{\begin{array}{c}3 x+6 y \leq 60 \\ 4 x+2 y \leq 32 \\ x \geq 0 \\ y \geq 0\end{array}\right.$

Para desenhar a região viável, o aluno faz a inserção de cada uma das restrições digitando as inequações na entrada de comandos do Geogebra. A Figura 5 traz a região associada a cada inequação do modelo (6), sendo a região viável obtida por meio da interseção entre a região de cada inequação. 


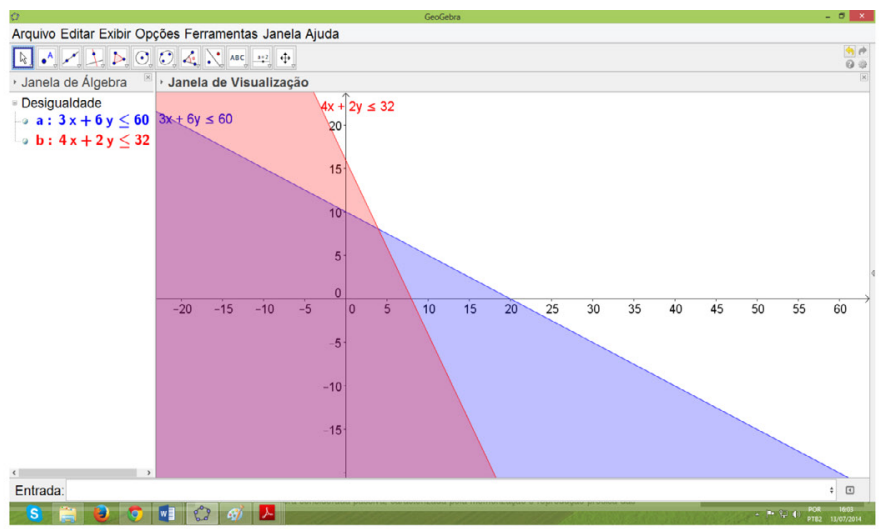

Figura 5: Geogebra para representar as inequações do modelo (6). Fonte: Elaborada pelo autor.

O próximo passo consiste em encontrar os pontos extremos da região viável. Para tanto, é preciso ir à barra de ferramentas do Geogebra e selecionar o botão que permite inserir pontos na zona gráfica. A ideia é que o aluno marque os pontos justamente nos extremos da região viável e, então, torna-se simples obter as coordenadas de cada um deles. Na Figura 6 está ilustrado o botão "Ponto", que o aluno precisa clicar para, em seguida, inseri-lo no respectivo local da zona gráfica associado aos vértices extremos (pontos $A, B, C$ e D).

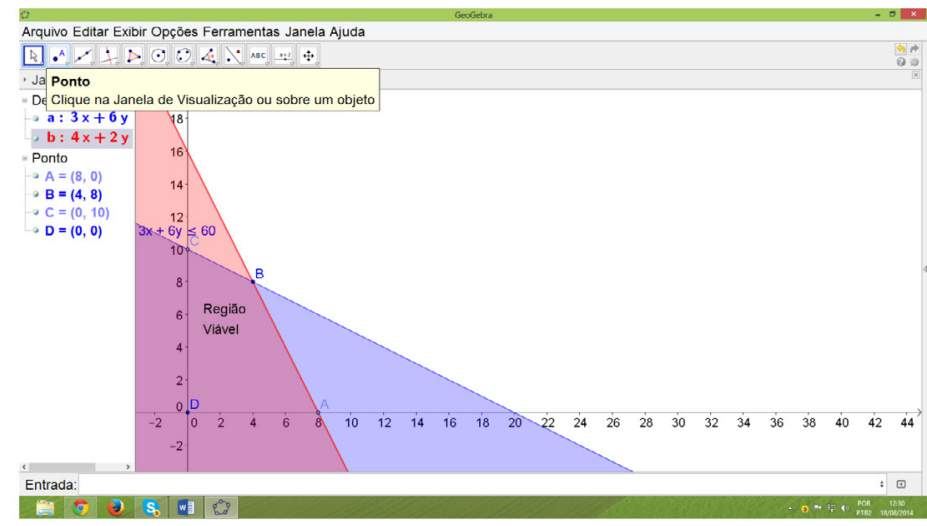

Figura 6: Usando o Geogebra para encontrar os pontos extremos do modelo (6). Fonte: Elaborada pelo autor.

Assim, com os pontos extremos, pode-se utilizar a substituição de pontos na função objetivo. No Geogebra, faz-se a utilização da planilha, que é acessada pelo botão exibir, conforme ilustra a Figura 7. 


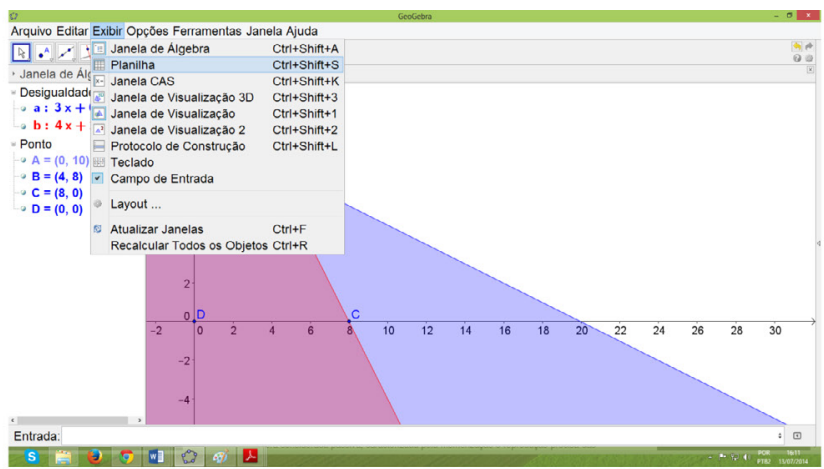

Figura 7: Geogebra apresentando a planilha de cálculo. Fonte: Elaborada pelo autor.

Ao acessar a planilha de cálculos, deve-se proceder com a substituição dos pontos encontrados na função objetivo. Para cada ponto, tem-se seu valor na função objetivo de acordo com a Figura 8.

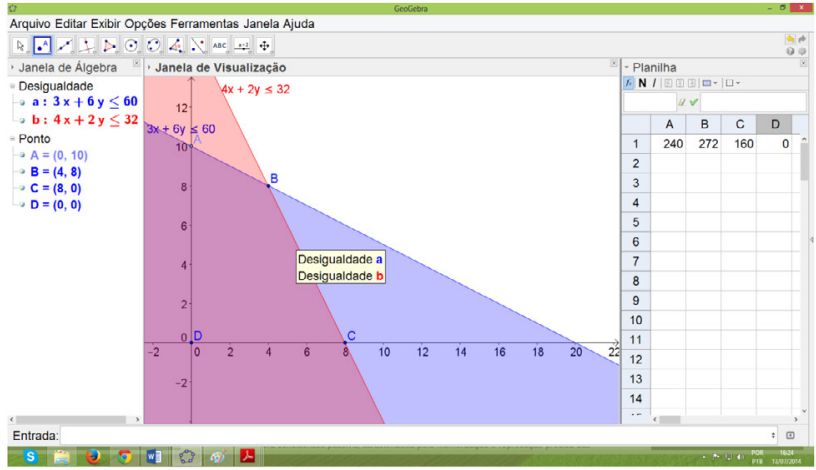

Figura 8: Geogebra mostrando o valor da função objetivo para o modelo (6). Fonte: Elaborada pelo autor.

Como o objetivo do modelo (6) é maximização, a solução ótima consiste no vértice de coordenadas $(x, y)=(4,8)$, que é representada pelo ponto $B$. Observe na Figura 8 que o valor da função objetivo nesse ponto é igual 272 , que é o máximo entre os todos.

\subsection{Uma Extensão para Problemas com Três Variáveis}

Aborda-se agora problemas de programação linear com três variáveis de decisão, que também podem ser resolvidos por meio do Geogebra 5.0 Beta Release. Assim, tem-se a região viável representada no espaço tridimensional. Parte-se para o uso do Geogebra por meio do seguinte modelo de programação linear, a saber: 
$\operatorname{Maximizar} \mathrm{z}=\mathrm{x}+\mathrm{y}+\mathrm{z}$

Sujeito a: $\left\{\begin{array}{l}x+2 y+z \leq 6 \\ 4 x+y+2 z \leq 8 \\ x \geq 0, y \geq 0, z \geq 0\end{array}\right.$

Existem três variáveis no modelo (7), portanto a região viável é um sólido em três dimensões. Para a visualização da região viável, busca-se, inicialmente, a intersecção entre as restrições e os eixos. Na primeira restrição, tem-se os pontos $A(6,0,0), B(0,3,0)$ e $C(0,0,6)$, que são obtidos anulando-se duas a duas as variáveis, e na segunda restrição, tem-se $\mathrm{D}(2,0$, $0), E(0,8,0)$ e $F(0,0,4)$. Em seguida, insere-se os pontos no Geogebra usando a linha de comandos e, com a função polígono, faz-se o desenho de cada uma dos planos obtendo a região viável ilustrada na Figura 9. Note que apenas o primeiro octante é considerado, uma vez que as variáveis possuem domínio não-negativo.

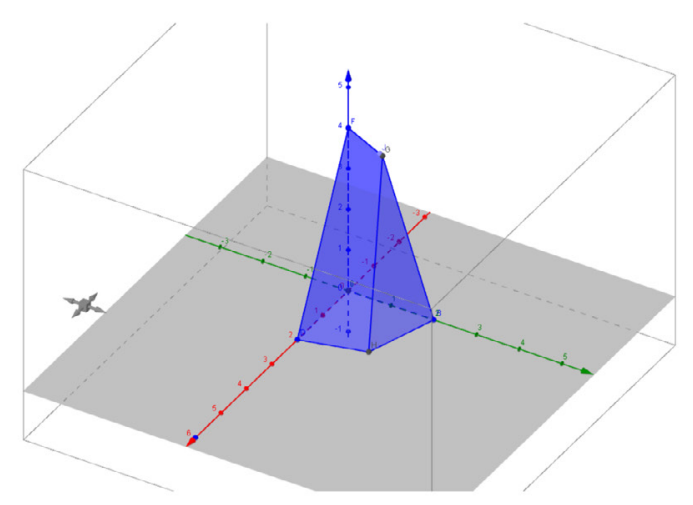

Figura 9: Região viável do modelo (7) formada pela interseção de suas restrições.

Fonte: Elaborada pelo autor.

Após obter a região viável do modelo (7), parte-se para a determinação dos pontos extremos. Neste caso, resta determinar apenas os pontos $\mathrm{G}$ e H. Em seguida, usando a linha de comandos, insere-se a função objetivo, atribuindo diferentes valores a ela, com o intuito de formar distintas curvas de nível (similar ao caso com duas variáveis). As curvas de nível correspondem a planos paralelos e o objetivo é encontrar o plano que traz o máximo valor para o problema. A Figura 10 mostra na cor azul os diferentes planos (curvas de nível) para a função objetivo, em que é possível perceber que o ponto extremo para a função é o $G$. 


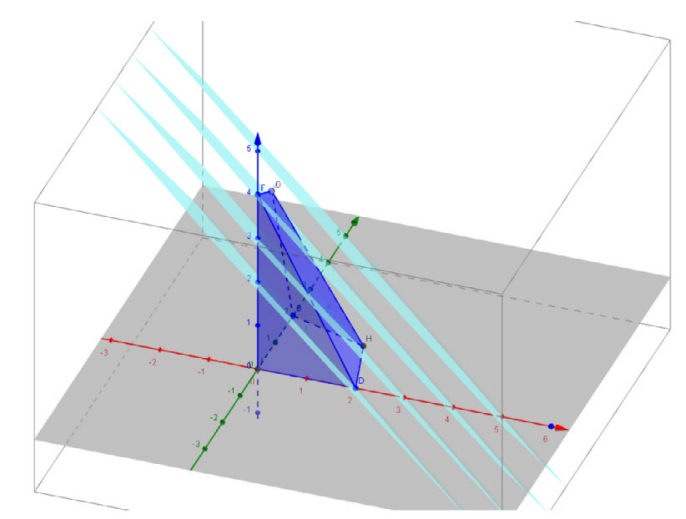

Figura 10: Representação da função objetivo na região viável do modelo (7).

Fonte: Elaborada pelo autor

Com o intuito de descobrir o valor da função objetivo no ponto $\mathrm{G}(0 ; 0,8 ; 3,6)$, faz-se a substituição na função objetivo e obtém-se o valor $z=4,4$. Observa-se que a teoria para resolver problemas de três variáveis continua a mesmo, ou seja, o ponto de solução ótima continua sendo um dos vértices extremos da região viável.

\section{Considerações Finais}

Por meio do presente trabalho foi possível verificar que a geometria passou e está passando por transformações na forma, nos conteúdos e na metodologia de ensino. $O$ professor precisa ficar atento a tais transformações e, assim, ser capaz de fornecer ao aluno um ensino-aprendizado mais adequado à realidade atual. Nesse contexto, a inserção de situações-problemas contextualizadas se torna muito importante, sendo este o propósito da programação linear no ensino médio.

A programação linear, trabalhada com duas ou três variáveis, permite que o aluno tenha uma relação contextualizada com a geometria analítica, uma vez que se trabalha a partir de situações-problemas retirados do cotidiano do aluno e, a partir disso, aplicam-se os conhecimentos geométricos. Existe ainda o uso do software matemático, Geogebra, ficando mais evidente as potencialidades para se trabalhar com os alunos usando diferentes técnicas e abordagens de ensino.

Por fim, a pesquisa realizada procura colocar de forma clara que a programação linear pode (e deve) ser ensinada no ensino médio, ainda mais, quando se usa um software matemático como sustentação metodológica.

Como proposta para trabalhos futuros, destaca-se a inserção desta proposta de abordagem da geometria analítica no ensino médio. Outra proposta consiste em trabalhar com o método simplex no ensino médio, porém, esta não deve ser uma tarefa trivial, uma vez que o currículo atual não abre margens para a inserção de novos conteúdos de forma direta. 


\section{Agradecimentos}

Os autores agradecem $\circ$ apoio financeiro recebido das agências $\mathrm{CNPq}$ (471351/2012-1) e FAPEG. 


\section{Teaching Analytic Geometry by using Linear Programming}

Abstract: Analytic Geometry has been, over the time, reason of much anxiety among high school students. From that anguish emerges the desire of make such subject accessible to any student and, then, the search for examples experienced by them becomes relevant. This work aims to facilitate the relationship of students with some of the topics in analytic geometry. Therefore, to use linear programming and a mathematical software become an important methodology, since arise some possibilities contributing to the achievement of the following objectives: to deal with modeling, solve real life problems, and interpret results in practical scenarios.

\section{Keywords: Linear programming; High school; Analytic geometry.}

\section{Referências bibliográficas}

ARENALES, M., et al. Pesquisa Operacional. 8 reimpressão. Rio de Janeiro: Campus, 2007.

BRASIL, Ministério da Educação. PCN + Ensino Médio: Orientações educacionais complementares aos parâmetros curriculares nacionais. Ciências da Natureza, Matemática e suas Tecnologias. Brasília: MEC, 2006.

DANTE, L. R. Coleção: Novo Ensino Médio. São Paulo: Ática, 2005.

DANTZIG, G. B. Linear Programming and Extensions. Princeton: Princeton University Press, 1963.

DEWEY, J. Vida e Educação. Tradução Anízio Teixeira. 6 ed. São Paulo: Melhoramentos, 1967.

HILLIER, F. S; LIEBERMAN, G. J. Introdução à pesquisa operacional. 9 ed. Porto Alegre: AMGH, 2013.

HOHENWARTER, M; HOHENWARTER, J. Ajuda Geogebra: Manual Oficial da Versão 3.2. 2009. 97f. Disponível em: http://www.geogebra.org/help/docupt_PT.pdf. Acesso em 07 de julho de 2014. 
PASSOS, E. J. P. F. Programação Linear como Instrumento da Pesquisa Operacional. São Paulo: Atlas, 2008.

REIS, G. L.; SILVA, V. V. Geometria Analítica. 2 ed. Rio de Janeiro: LTC, 1996.

SILVA, C. X.; BARRETO FILHO, B. Matemática aula por aula. Vol. Único. São Paulo: FTD, 2000.

YOSHIDA, L. K. Programação Linear, Métodos Quantitativos. São Paulo: Editora Atual LTDA, 1987. 